\title{
Investigating Reaction Wheel Configuration and Control Law Pairings for CubeSats in the Presence of Faults
}

\author{
Andrew Newton, Elyse Hill, S. Andrew Gadsden ${ }^{1}$, Mohammad Biglarbegian, and Simon Yang \\ ${ }^{1}$ College of Engineering and Physical Sciences \\ University of Guelph, Guelph, ON, Canada \\ Emails: anewto03, ehill06, gadsden, mbiglarb, and syang@uoguelph.ca
}

\begin{abstract}
In this paper, the attitude control of a model CubeSat was simulated using the nonlinear control techniques of feedback linearization and sliding mode control. The dynamic model of the CubeSat was derived based on a reaction wheel actuation system, which was presented in two configurations. The performance of the controllers on the CubeSat were compared in a nominal mode of operation and a faulty mode of operation. The benefit to combining a controller with a specific reaction wheel configuration was also evaluated based on the root mean squared error of the CubeSat attitude and angular velocities. Results show that a sliding mode controller performs better than a feedback linearization controller with a pyramid configuration in a fault mode. However, using attitude error as the evaluation metric for any potential controller-configuration relationship is tenable and other evaluation parameters should be considered
\end{abstract}

Keywords-CubeSats; control theory; fault prediction; nonlinear system modeling; spacecraft; attitude control

\section{INTRODUCTION}

The attitude control system, which determines the ability to achieve and maintain a desired orientation, is a mission critical subsystem in any spacecraft. Reaction wheels are a popular type of attitude control actuator that offer a high degree of pointing accuracy and three degree of freedom control authority. As of late, increased pointing accuracy is desired of small satellites, such as CubeSats. Typical control architectures on these small spacecraft currently employ magnetorquers, which function in low earth orbit, at the cost of less accurate pointing. Implementation of reaction wheels on CubeSats has been challenging due to size and weight constraints, as well as the presence of faults upon launch [1]. Therefore, investigation into reaction wheel performance on CubeSat attitude control is warranted.

Satellites are often equipped with three reaction wheels for three axis control, with additional wheels added for redundancy. Various wheel configurations exist and are the focus of several studies, including [2], which studied reaction wheel configurations in three- and four- wheel schemes, in an attempt to optimize reaction wheel orientation for minimal power consumption. As moving mechanisms, reaction wheels are subject to actuator failure, including loss of efficiency, float, or lock-in-place faults [3]. Research by [4], in which multiple fourwheel configurations were subjected to float failures, seems to suggest that some configurations are more robust to failures than others. Thus, pairing certain reaction wheel orientations with selected control methods seems beneficial.

This study will investigate the benefits of controller and reaction wheel configuration pairings by evaluating controller performance on a model CubeSat with two reaction wheel configurations. Two nonlinear control techniques will be employed: sliding mode control and feedback linearization control. Although proportional-derivative controllers are conventionally employed for attitude control, nonlinear control techniques are applied to maintain the nonlinearity of spacecraft dynamics [5]. Sliding mode control has been widely used for attitude control due to its robustness in the face of uncertainty [6] [7] [8] [9]. Feedback linearization has also found application in attitude control in the works of [10] [11] [12]. Controller performance will be evaluated based on attitude errors. The goal of the controller is attitude regulation to the identity quaternion in a no-fault scenario and with a float fault scenario applied on one reaction wheel.

\section{Mathematical Model of Satellite System}

\section{A. Satellite Kinematics and Dynamics}

The attitude of a satellite can be represented by the quaternion, $\mathbf{q}=\left[\begin{array}{llll}\mathrm{q}_{1} & \mathrm{q}_{2} & \mathrm{q}_{3} & \mathrm{q}_{4}\end{array}\right]^{\mathrm{T}}$, which has vector part $\mathbf{q}_{1: 3}$ and scalar part $\mathrm{q}_{4}$. This parameterization is used to avoid singularities that would be present using Euler angle notation. A rotation from the inertial frame to the body frame of a satellite can be described by a quaternion parameterized attitude matrix:

$$
\mathrm{A}(\mathbf{q})=\left(\mathrm{q}_{4}^{2}-|| \mathbf{q}_{1: 3}||^{2}\right) \mathrm{I}_{3}+2 \mathbf{q}_{(1: 3)} \mathbf{q}_{1: 3}^{\mathrm{T}}-2 \mathrm{q}_{4}\left[\mathbf{q}_{1: 3} \times\right]
$$

Where a skew-symmetric matrix is described as: 


$$
[\mathbf{y} \times]=\left[\begin{array}{ccc}
0 & -y_{3} & y_{2} \\
y_{3} & 0 & -y_{1} \\
-y_{2} & y_{1} & 0
\end{array}\right]
$$

The kinematic and dynamic equations of a rigid body spacecraft with reaction wheels in the absence of external disturbances can be defined as:

$$
\begin{gathered}
\dot{\mathbf{q}}=\frac{1}{2} \boldsymbol{\Omega}\left(\boldsymbol{\omega}_{\mathbf{b}}\right) \mathbf{q}, \\
\dot{\boldsymbol{\omega}}_{\mathrm{b}}=\mathbf{I}_{\mathbf{b}}^{\mathbf{- 1}}\left[\mathbf{T}_{\mathbf{c}}-\left[\boldsymbol{\omega}_{\mathbf{b}} \times\right]\left(\mathbf{I}_{\mathbf{b}} \boldsymbol{\omega}_{\mathbf{b}}+\mathbf{h}_{\mathbf{w}}\right)\right] .
\end{gathered}
$$

Where $\omega_{b}$ is the angular velocity of the satellite body in the body frame, $\mathbf{I}_{\mathbf{b}}$ is the moment of inertia of the satellite body represented as a $3 \times 3$ matrix, $\mathbf{h}_{\mathbf{w}}$ is the reaction wheel angular momentum vector with respect to the body frame, the control torque input is $\mathbf{T}_{\mathbf{c}}=\dot{\mathbf{h}}_{\mathbf{w}}$, and the operation $\boldsymbol{\Omega ( y )}$ equals

$$
\boldsymbol{\Omega}(\mathbf{y})=\left[\begin{array}{cc}
-\left[y^{x}\right] & \mathbf{y} \\
-\mathbf{y}^{\mathbf{T}} & 0
\end{array}\right] \text {. }
$$

Equations 3 and 4 can be expressed in state space form as:

$$
\begin{aligned}
& \dot{\mathbf{x}}=\mathbf{f}\left(\mathbf{x}, \mathbf{T}_{\mathbf{c}}\right)=\mathbf{f}(\mathbf{x})+\mathbf{g}(\mathbf{x}) \mathbf{T}_{\mathbf{c}} \\
& \mathbf{z}=\mathbf{h}(\mathbf{x})
\end{aligned}
$$

Where the states are defined $\mathbf{x}=\left[\begin{array}{ll}\mathbf{q} & \boldsymbol{\omega}_{\mathbf{b}}\end{array}\right]^{\mathrm{T}}$.

\section{B. Reaction Wheels}

Satellites are often equipped with more than three reaction wheels for redundancy. Wheel configuration changes how the wheel torque is distributed to the spacecraft body. This is accounted for with a distribution matrix, $\mathbf{L}$, which maps the torque from the wheel frame into the satellite body frame. This can be expressed as:

$$
\dot{\mathbf{h}}_{\mathbf{w}}=\mathbf{L} \dot{\mathbf{h}}_{\mathbf{w}}^{\mathrm{W}}
$$

where the superscript $\mathrm{W}$ represents the wheel coordinate frame. Two separate wheel configurations are considered in this paper: the NASA standard and the Pyramid configuration. The distribution matrix for the NASA standard configuration is:

$$
\mathbf{L}=\left[\begin{array}{llll}
1 & 0 & 0 & \alpha \\
0 & 1 & 0 & \beta \\
0 & 0 & 1 & \gamma
\end{array}\right],
$$

which represents a wheel aligned along each body axis and a fourth wheel placed skew to each axis (Fig. 1). Here, $\alpha, \beta$, and $\gamma$ are user specified values that follow the constraint $\alpha^{2}+\beta^{2}+\gamma^{2}=1$. The Pyramid configuration distribution matrix is:

$$
\mathbf{L}=\left[\begin{array}{cccc}
\mathrm{a} & -\mathrm{a} & 0 & 0 \\
\mathrm{~b} & \mathrm{~b} & \mathrm{c} & \mathrm{c} \\
0 & 0 & \mathrm{~d} & -\mathrm{d}
\end{array}\right]
$$

which represents wheels skew to all primary axes, with preferential alignment to the $\mathrm{Y}$-axis (Fig. 2). The values a, b, c and $\mathrm{d}$ follow the constraints $\mathrm{a}^{2}+\mathrm{b}^{2}=\mathrm{c}^{2}+\mathrm{d}^{2}=1$ and indicate the degree of skew of each wheel, represented by the angle $\beta$ in Fig. 2. Reaction wheels are subject to several failure modes. In this paper, the float failure, where actuation is zero, is explored. A fault can be modeled mathematically as [13]:

$$
T_{a}=T_{c}+\sigma\left[T_{i}-T_{c}\right]
$$

where $\mathbf{T}_{\mathbf{a}}$ is the torque actuated by the wheels, $\mathbf{T}_{\mathbf{c}}$ is the torque commanded by the control law, $\boldsymbol{\sigma}=\operatorname{diag}\left(\sigma_{1} \sigma_{2} \sigma_{3} \sigma_{4}\right)$ is the actuator failure indicator, and $\mathbf{T}_{\mathbf{i}}$ is the actuator failure.

\section{Tracking Error Dynamics}

The tracking error for the system can be defined as the difference between the actual and desired values:

$$
\begin{gathered}
q_{e}=q \otimes q_{d}^{-1}, \\
\omega_{e}=\omega_{b}-\delta A \omega_{d} .
\end{gathered}
$$

where $\mathbf{q}$ is the actual quaternion, $\mathbf{q}_{\mathbf{d}}$ is the desired quaternion, $\omega_{b}$ is the actual angular velocity of the body, $\boldsymbol{\omega}_{\mathrm{d}}$ is the desired angular velocity of the body, and $\delta \mathrm{A}=\mathrm{A}(\mathbf{q}) \mathrm{A}_{\mathrm{d}}^{\mathrm{T}}\left(\mathbf{q}_{\mathbf{d}}\right)$ is the

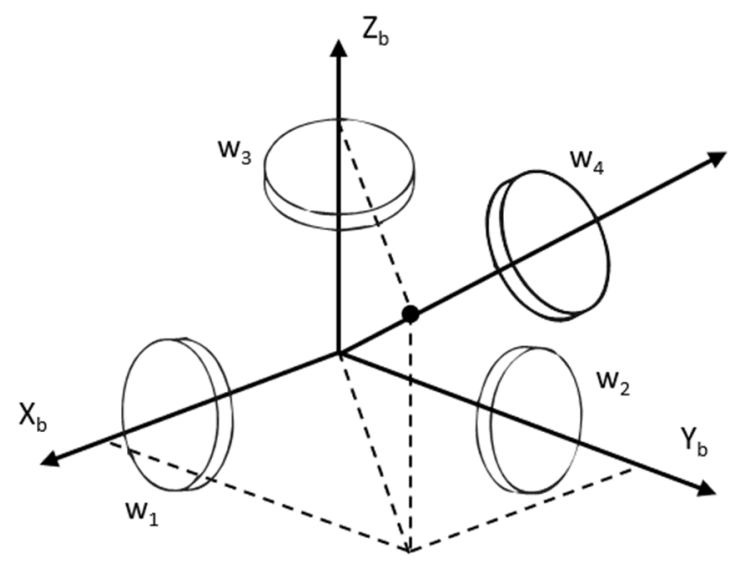

Figure 1. NASA Standard configuration for redundant reaction wheels [6].

quaternion parameterized attitude error matrix that resolves the angular velocity error in body frame coordinates. The operator $\mathbf{q} \otimes$ represents:

$$
\mathbf{q} \otimes=\left[\begin{array}{cc}
\mathrm{q}_{4} \mathbf{I}_{3}-\left[\mathbf{q}_{1: 3} \times\right] & \mathbf{q}_{1: 3} \\
-\mathbf{q}_{1: 3}^{\mathbf{T}} & \mathrm{q}_{4}
\end{array}\right]
$$

A tracking controller's goal is to follow a desired reference trajectory such that $\mathbf{q}_{\mathbf{e}, 1: 3} \rightarrow \mathbf{0}$ and $\boldsymbol{\omega}_{\mathbf{e}} \rightarrow \mathbf{0}$. Regulation is a special form of tracking where the goal is to drive the quaternion to identity, $\mathbf{q}=\left[\begin{array}{llll}0 & 0 & 0 & 1\end{array}\right]^{\mathrm{T}}$, and drive the body angular velocities to zero, $\boldsymbol{\omega}_{\mathbf{b}}=\left[\begin{array}{lll}0 & 0 & 0\end{array}\right]^{\mathrm{T}}[6]$. In this case, the error dynamics of Eqs. 11 and 12 reduce to:

$$
\begin{gathered}
\dot{\mathbf{q}}_{\mathrm{e}}=\frac{1}{2} \boldsymbol{\Omega}\left(\boldsymbol{\omega}_{\mathbf{b}}\right) \mathbf{q}_{\mathrm{e}} \\
\dot{\boldsymbol{\omega}}_{\mathrm{e}}=\mathbf{I}_{\mathbf{b}}^{\mathbf{- 1}}\left[\mathbf{T}_{\mathbf{c}}-\left[\omega_{\mathbf{b}} \times\right]\left(\mathbf{I}_{\mathbf{b}} \omega_{\mathbf{b}}+\mathbf{h}_{\mathrm{w}}\right)\right]
\end{gathered}
$$




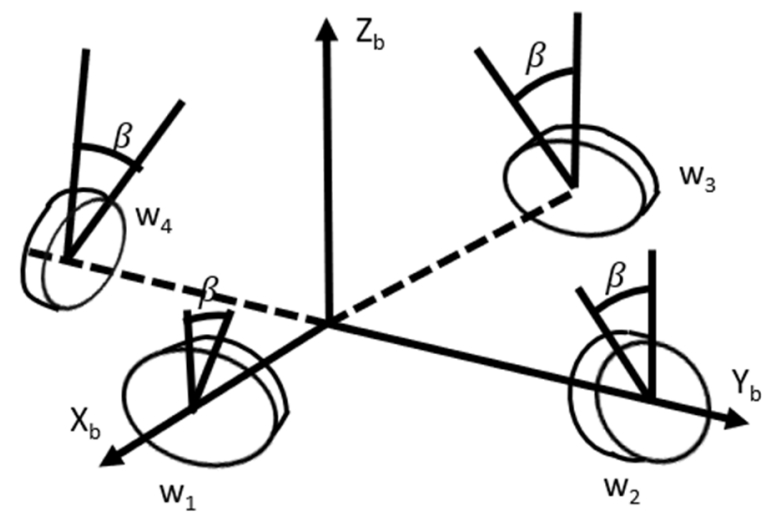

Figure 2. Pyramid configuration for redundant reaction wheels [14].

\section{CONTROLler Design}

\section{A. Feedback Linearization Controller}

Feedback linearization requires a system of governing equations in the form

$$
\dot{\mathbf{x}}=\mathbf{f}(\mathbf{x})+\mathbf{b}(\mathbf{x}) \cdot \mathbf{u}
$$

where $\mathbf{f}(\mathbf{x})$ and $\mathbf{b}(\mathbf{x})$ are nonlinear functions of the state variable and time and $\mathbf{u}$ is the control input. $\mathbf{u}$ is designed such that $\mathbf{f}(\mathbf{x})$ and $\mathbf{b}(\mathbf{x})$ are eliminated, therefore:

$$
u=\frac{1}{b(x)}(-f(x)+v)
$$

where $\mathbf{v}$ is an auxiliary variable and is designed to impose stability on the system. A common design for $\mathbf{v}$ is modeled using error dynamics:

$$
\mathbf{v}=\mathbf{x}_{\mathrm{ref}}^{\mathbf{n}}-\mathrm{K}_{1} \mathbf{e}^{\mathrm{n}-1}-\ldots-\mathrm{K}_{(\mathrm{n}-1)} \mathbf{e} .
$$

Applied to the satellite dynamic model this yields:

$$
\mathbf{v}=\dot{\boldsymbol{\omega}}_{\mathbf{d}}-\mathrm{k}_{\mathrm{d}} \boldsymbol{\omega}_{\mathrm{e}}-\mathrm{k}_{\mathrm{p}} \operatorname{sign}\left(\mathrm{q}_{\mathrm{e}, 4}\right) \mathbf{q}_{\mathrm{e}, 1: 3}
$$

where $\mathrm{k}_{\mathrm{p}}$ and $\mathrm{k}_{\mathrm{d}}$ are proportional and derivative feedback gains, respectively. The $\operatorname{sign}\left(q_{e, 4}\right)$ term is added to ensure that the shortest route possible is taken from the current to the desired quaternion [6]. Using Eq. 19, the feedback linearized control torque is:

$$
\begin{aligned}
& \mathbf{u}=\mathbf{I}_{\mathbf{b}}\left[-\mathbf{I}_{\mathbf{b}}^{-1}\left(\left[-\boldsymbol{\omega}_{\mathbf{b}} \times\right]\left(\mathbf{I}_{\mathbf{b}} \boldsymbol{\omega}_{\mathbf{b}}+\mathbf{h}_{\mathbf{b}}\right)\right)+\dot{\boldsymbol{\omega}}_{\mathbf{d}}-\mathrm{k}_{\mathrm{d}} \boldsymbol{\omega}_{\mathbf{e}}\right. \\
& \left.\quad-\mathrm{k}_{\mathrm{p}} \operatorname{sign}\left(\mathrm{q}_{\mathrm{e}, 4}\right) \mathbf{q}_{\mathrm{e}, 1: 3}\right]
\end{aligned}
$$

\section{B. Sliding Mode Controller}

A sliding mode controller is designed using the quaternion error. The sliding surface is:

$$
\mathbf{S}=\left(\frac{\mathrm{d}}{\mathrm{dt}}+\lambda\right) \mathbf{e}=\dot{\mathbf{e}}+\lambda \mathbf{e}=\boldsymbol{\omega}_{\mathbf{e}}+\lambda \mathbf{q}_{\mathbf{e}, 1: 3} .
$$

Differentiating the sliding surface with respect to time results in:

$$
\dot{\mathbf{S}}=\dot{\boldsymbol{\omega}}_{\mathbf{e}}+\lambda \dot{\mathbf{q}}_{\mathbf{e}, 1: 3} \text {. }
$$

To solve for the estimated control torque, $\widehat{\mathbf{T}}_{\mathbf{c}}$, Eq. 15 is plugged into Eq. 22 and then the equation is set equal to zero, producing:

$$
\widehat{\mathbf{T}}_{\mathbf{c}}=\left[\boldsymbol{\omega}_{\mathbf{b}} \times\right]\left(\mathbf{I}_{\mathbf{b}} \boldsymbol{\omega}_{\mathbf{b}}+\mathbf{h}_{\mathbf{w}}\right)-\mathbf{I}_{\mathbf{b}} \lambda \dot{\mathbf{q}}_{\mathbf{e}, 1: 3}
$$

The total input, $\mathbf{T}_{\mathbf{c}}$, is found by adding a discontinuous term across the sliding surface:

$$
\mathbf{T}_{\mathbf{c}}=\widehat{\mathbf{T}}_{\mathbf{c}}-\mathbf{I}_{\mathbf{b}} \mathbf{K} \operatorname{sign}(\mathbf{S})
$$

where $\mathbf{K}$ is a diagonal $3 \times 3$ matrix.

\section{Simulations AND Results}

This paper modified an example from [6], which performed a regulation attitude maneuver, and implemented it in MATLAB $^{\circledR}$. Note that the identity quaternion is equivalent to an attitude in Euler angles of $\left[\begin{array}{lll}\phi & \theta & \psi\end{array}\right]^{\mathrm{T}}=\left[\begin{array}{lll}0 & 0 & 0\end{array}\right]^{\mathrm{T}}$ degrees. System parameters and initial conditions are provided in Tab. 1. Values of the inertia matrix and maximum torque are based on those found in [15] for a $3 \mathrm{U}$ CubeSat with Microwheel reaction wheels. The initial attitude is set to $\left[\begin{array}{lll}\phi_{0} & \theta_{0} & \psi_{0}\end{array}\right]^{\mathrm{T}}=\left[\begin{array}{lll}60 & 20 & 40\end{array}\right]^{\mathrm{T}}$ degrees while the initial values of body angular velocity remain unchanged from [6]. Gain values were tuned manually based on the nominal operation of the system. They remained unchanged for failure testing.

Two operating modes were considered: nominal and float fault. Nominal mode was characterized by $\boldsymbol{\sigma}=\operatorname{diag}\left(\mathbf{0}_{\mathbf{4}}\right)$ while in fault mode $\boldsymbol{\sigma}=\operatorname{diag}(1,0,0,0)$, indicating a float failure of one reaction wheel (Wheel 1). In both modes, $\mathbf{T}_{\mathbf{i}}=\left[\begin{array}{llll}0 & 0 & 0 & 0\end{array}\right]^{\mathrm{T}}$. Simulation time is equivalent to $\mathrm{t}=5 \mathrm{~min}$.

The root mean squared errors (RMSE) for the roll, pitch, yaw, and corresponding body angular velocities were used to evaluate controller performance. Tab. 2 displays these values for both configurations in nominal mode. Figs. 3-6 display the attitude maneuvers in nominal mode for both controllers and both configurations. Similarly, metrics for fault mode are presented in Tab. 3 and graphical representations of the fault mode responses are represented in Figs. 7-10. The attitude is displayed in Euler angles for ease of presentation, analysis, and intuition.

TABLE I. SYSTEM PARAMETERS

\begin{tabular}{|c|c|}
\hline Parameter & Value \\
\hline $\mathbf{I}_{\mathbf{b}}$ & {$\left[\begin{array}{ccc}0.0056 & 0 & 0 \\
0 & 0.026 & 0 \\
0 & 0 & 0.0026\end{array}\right] \mathrm{kgm}^{2}$} \\
\hline$\lambda$, & 0.07 \\
$\mathbf{K}$ & $0.007 \times \mathrm{I}_{3}$ \\
\hline $\mathrm{K}_{\mathrm{p}}$ & 4 \\
$\mathrm{~K}_{\mathrm{d}}$ & $\frac{1}{\sqrt{3}}$ \\
\hline$\alpha, \beta, \gamma$ & $\frac{1}{\sqrt{2}}$ \\
\hline $\mathrm{a}, \mathrm{b}, \mathrm{c}, \mathrm{d}$ & $4.0 \mathrm{mNm}$ \\
\hline $\mathbf{T}_{\mathbf{c}, \max }$ & {$\left[\begin{array}{ccc}60 & 20 & 40\end{array}\right]^{\mathrm{T}} \mathrm{deg}$} \\
\hline$\left[\phi_{0}, \theta_{0}, \psi_{0}\right]^{\mathrm{T}}$ & {$\left[\begin{array}{ccc}0.57 & 0.57 & 0.57\end{array}\right]^{\mathrm{T}} \mathrm{deg} / \mathrm{s}$} \\
\hline $\boldsymbol{\omega}_{\mathbf{b}, \mathbf{0}}$ & 3 \\
\hline
\end{tabular}


TABLE II. NOMINAL Mode Performance

\begin{tabular}{|c|c|c|c|c|c|c|}
\hline \multicolumn{7}{|c|}{ Pyramid Configuration } \\
\hline & \multicolumn{3}{|c|}{ Attitude Error (deg) } & \multicolumn{3}{|c|}{$\begin{array}{c}\text { Angular Velocity Error } \\
\text { (deg/s) }\end{array}$} \\
\hline Controller & $\phi$ & $\theta$ & $\psi$ & $\omega_{\mathrm{x}}$ & $\omega_{\mathrm{y}}$ & $\omega_{\mathrm{z}}$ \\
\hline FL & 3.33 & 2.78 & 1.99 & 1.20 & 1.93 & 3.61 \\
\hline SMC & 14.5 & 6.56 & 9.18 & 0.189 & 0.280 & 0.374 \\
\hline \multicolumn{7}{|c|}{ NASA Standard Configuration } \\
\hline & \multicolumn{3}{|c|}{ Attitude Error (deg) } & \multicolumn{3}{|c|}{$\begin{array}{c}\text { Angular Velocity Error } \\
(\mathrm{deg} / \mathrm{s})\end{array}$} \\
\hline Controller & $\phi$ & $\boldsymbol{\theta}$ & $\psi$ & $\omega_{\mathrm{x}}$ & $\omega_{\mathrm{y}}$ & $\omega_{\mathrm{z}}$ \\
\hline FL & 3.35 & 2.84 & 2.0 & 1.08 & 1.97 & 3.59 \\
\hline SMC & 14.5 & 6.56 & 9.18 & 0.189 & 0.280 & 0.374 \\
\hline
\end{tabular}

Both controllers perform the same across wheel configurations in the nominal mode, i.e. performance is identical in either configuration. This can be seen graphically in Figs. $3 \& 5$ and also Figs. $4 \& 6$, where the performances appear identical regardless of configuration, suggesting no benefit in application of one controller over another for either configuration. The feedback linearized controller (FLC) outperforms the sliding mode controller (SMC) in terms of attitude error but underperforms in terms of angular velocity error based on the values in Tab. 2. Further, the FLC settles much sooner than the SMC, at less than 1min compared to around $2 \mathrm{~min}$.

When the fault is present, fewer similarities are present across the configurations. Failing Wheel 1 corresponds to a different impact based on the configurations discussed previously. In the NASA standard configuration, this causes the wheel aligned on the $\mathrm{X}$-axis to lose control authority. For this orientation, the RMSE values for both controllers are still comparable to the nominal condition. The similarity in performance is because Wheel 4 allows the CubeSat to maintain 3-axis control.

TABle III. Fault Mode Performance

\begin{tabular}{|c|c|c|c|c|c|c|}
\hline \multicolumn{7}{|c|}{ Pyramid Configuration } \\
\hline & \multicolumn{3}{|c|}{ Attitude Error (deg) } & \multicolumn{3}{|c|}{$\begin{array}{c}\text { Angular Velocity Error } \\
(\mathrm{deg} / \mathrm{s})\end{array}$} \\
\hline Controller & $\phi$ & $\boldsymbol{\theta}$ & $\psi$ & $\omega_{\mathrm{x}}$ & $\omega_{\mathrm{y}}$ & $\omega_{\mathrm{z}}$ \\
\hline FL & 350.8 & 11.4 & 353.5 & 21.8 & 4.51 & 21.4 \\
\hline SMC & 16.2 & 7.63 & 24.4 & 0.606 & 0.409 & 0.201 \\
\hline \multicolumn{7}{|c|}{ NASA Standard Configuration } \\
\hline & \multicolumn{3}{|c|}{ Attitude Error (deg) } & \multicolumn{3}{|c|}{$\begin{array}{c}\text { Angular Velocity Error } \\
(\mathrm{deg} / \mathrm{s})\end{array}$} \\
\hline Controller & $\phi$ & $\boldsymbol{\theta}$ & $\psi$ & $\omega_{\mathrm{x}}$ & $\omega_{\mathrm{y}}$ & $\omega_{\mathrm{z}}$ \\
\hline FL & 3.86 & 2.52 & 11.7 & 6.77 & 1.76 & 4.40 \\
\hline SMC & 14.4 & 6.80 & 11.51 & 0.175 & 0.30 & 0.358 \\
\hline
\end{tabular}

Numerically, the controllers follow the same trend as nominal mode, but the FLC's RMSE for the angular velocities has increased while the SMC's values are approximately the same. This response indicates that, despite redundancy, the FLC is more sensitive to the failure of Wheel 1 than the SMC. FLC's sensitivity is displayed in Fig. 7, where it has high initial attitude and angular velocity errors before settling at around $1.5 \mathrm{~min}$. Though it settled faster than the SMC, as seen in Fig. 8 the SMC acts without large initial errors for attitude or angular velocity, providing a smoother attitude maneuver. The smoothness of the SMC is not surprising, however, given that it is known for being robust in the presence of disturbances.

The greatest difference in performance is seen in the controllers' fault mode pyramid configuration RMSE values. The SMC significantly outperforms the FLC, which has RMSE values nearly one hundred times its nominal mode values in roll and yaw. These are indicative of the fact these values fail to regain the target attitude as displayed in Fig. 9. Though the SMC sees some increase in its RMSE values, it still maintains error values similar to its nominal case. Failing Wheel 1 for the pyramid configuration affects control of the roll and pitch axes. Given the data, it seems the FLC is more susceptible to this failure than the SMC. This can be visualized in the figures, where again the FLC has large initial errors prior to settling whereas the SMC does not. Though the FLC settles in its angular velocity at around $1 \mathrm{~min}$, sooner than the $\mathrm{SMC}$ at around $3 \mathrm{~min}$, again the SMC provides a smoother attitude maneuver to the desired states than the FLC.
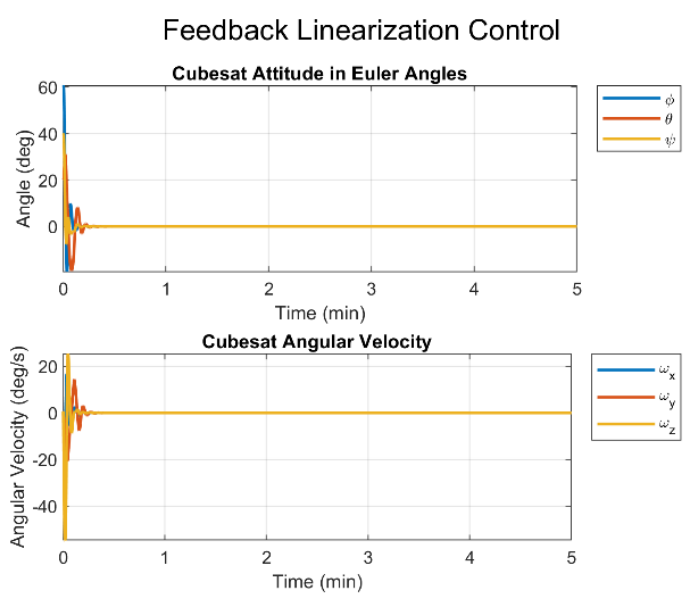

Figure 3. Attitude Error with FLC applied to NASA Standard Configuration (Nominal Mode) 
Sliding Mode Control

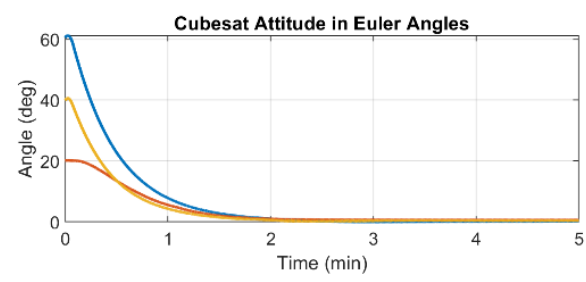

Cubesat Angular Velocity

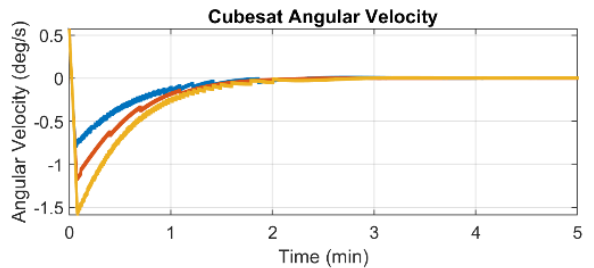

Figure 4. Attitude Error with SMC applied to NASA Standard Configuration (Nominal Mode)
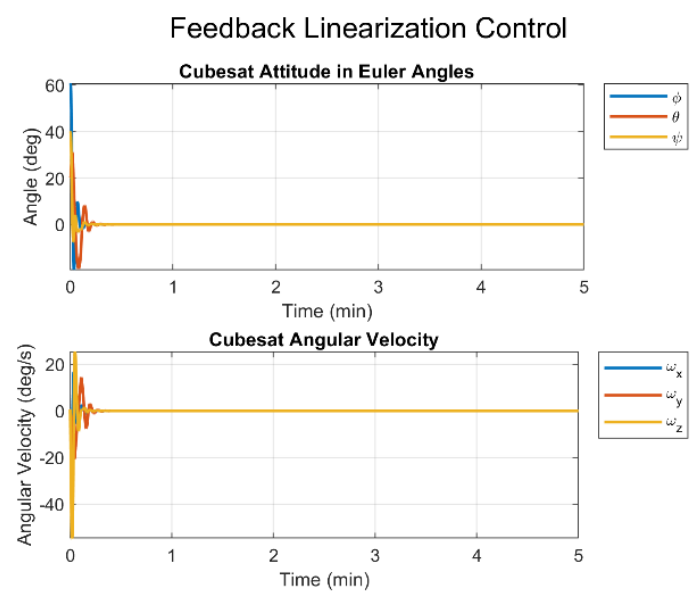

Figure 5. Attitude Error with FLC applied to Pyramid Configuration (Nominal Mode)

\section{Sliding Mode Control}

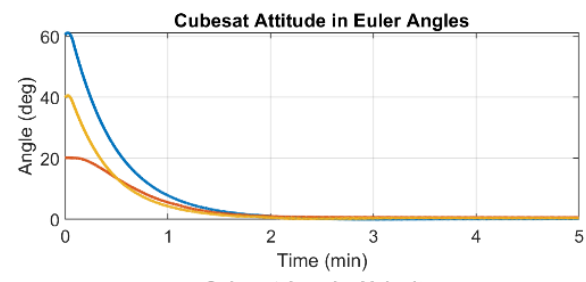

Cubesat Angular Velocity

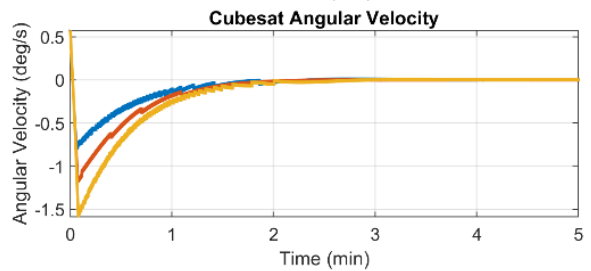

Figure 6. Attitude Error with SMC applied to Pyramid Configuration (Nominal Mode)
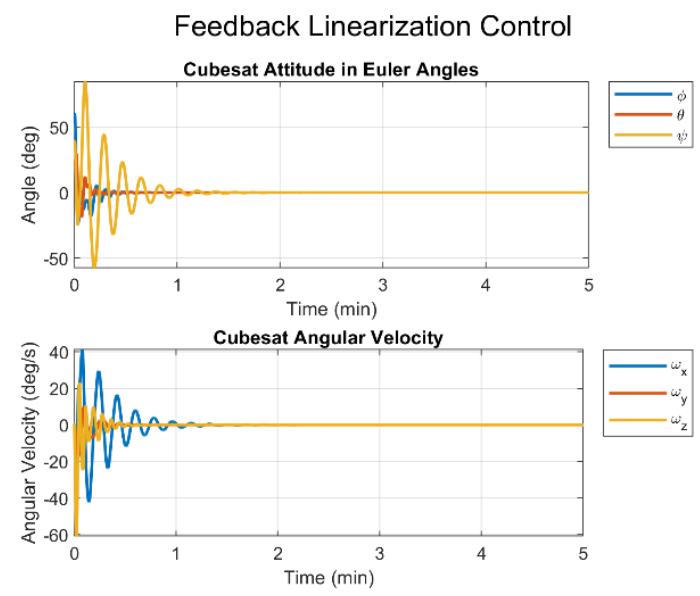

Figure 7. Attitude Error with FLC applied to NASA Standard Configuration (Fault Mode)
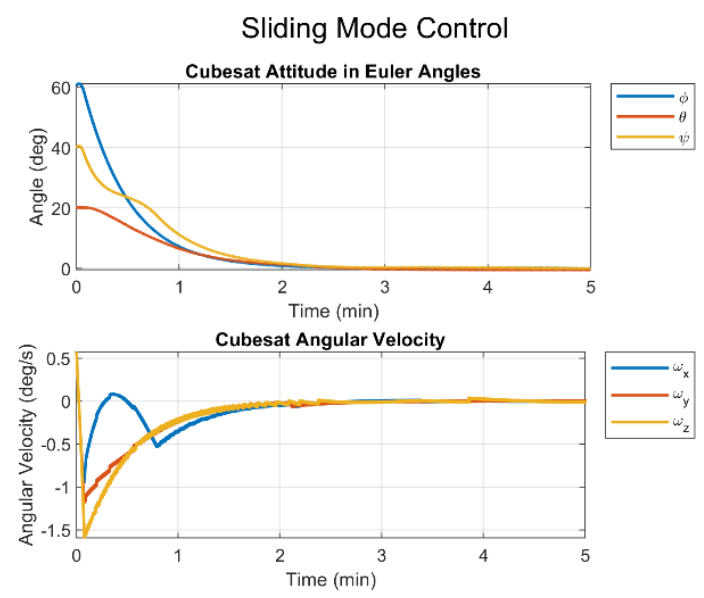

Figure 8. Attitude Error with SMC applied to NASA Standard Configuration (Fault Mode)
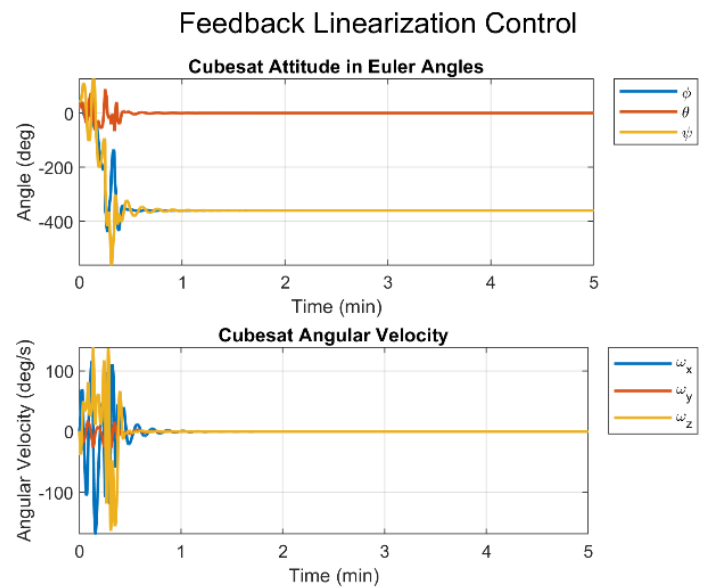

Figure 9. Attitude Error with FLC applied to Pyramid Configuration (Fault Mode) 
Sliding Mode Control
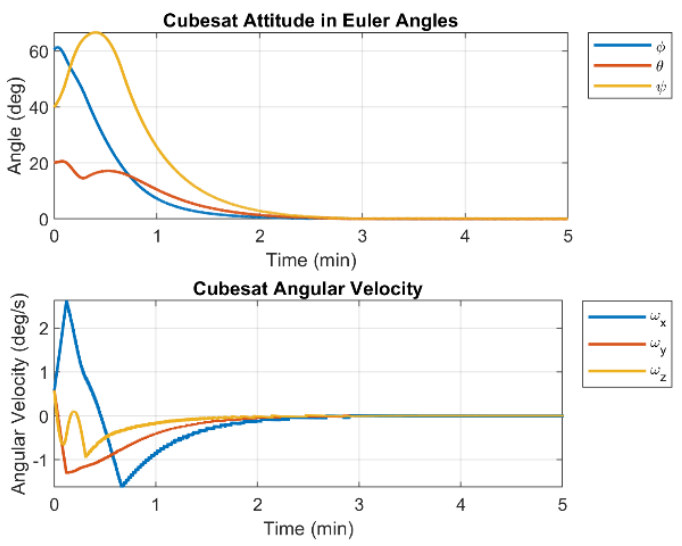

Figure 10. Attitude Error with SMC applied to Pyramid Configuration (Fault Mode)

\section{CONCLUSIONS}

This paper investigated the application of two nonlinear controllers to a model CubeSat bearing two separate reaction wheel configurations. The performance of the controllers was evaluated in the absence and presence of a float fault in an attempt to determine any benefit to pairing specific controllers and wheel configurations. In terms of error, the FLC and SMC performed identically in the absence of faults, with the FLC responding faster than the SMC by way of settling time. In this case, choice of controller and accompanying configuration made no difference in performance. In the presence of a float fault, however, controller-configuration pairing was significant on performance. This is best highlighted by the FLC's performance in pyramid configuration, where it failed to achieve the target attitude and had large initial attitude errors when compared to the SMC. However, because the SMC is known to be robust, it is difficult to conclude that there is a direct benefit to pairing specific controllers with reaction wheel configurations using this evaluation. Future explorations of a potential relationship between controller and configuration could examine power consumption, control effort, rise time, settling time, and steady state errors as a more indicative evaluation metric.

\section{REFERENCES}

[1] L. He, W. Ma, P. Guo and T. Sheng, "Developments of attittude determination and control system microsats: A survey," Proceedings of the Institution of Mechanical Engineers. Part I: Journal of Systems and Control Engineering, no. January, 2020.

[2] Z. Ismail and R. Varatharajoo, "A study of reaction wheel configurations for a 3-axis satellite attitude control," Advances in Space Research, vol. 45, no. 6, pp. 750-759, 2010.

[3] B. Jung, Y. Kim and C. Ha, "Fault tolerant flight control system design using a multiple model adaptive controller," Proceedings of the Institution of Mechanical Engineers, Part G: Journal of Aerospace Engineering, vol. 223, no. 1, pp. 39-50, 2009.

[4] I. Kök, "Comparison and Analysis of Attitude Control Systems of a Satellite Using Reaction Wheel Actuators," 2012.

[5] S. A. Gadsden, "An Adaptive PID Controller Based on Bayesian Theory," in ASME Dynamic Systems and Control Conference, Tysons Corner, Virginia, 2017.

[6] F. L. Markley and J. Crassidis, Space Technology Library Fundamentals of Spacecraft Attitude Determination and Control, New York: Springer, 2014.

[7] Y.-P. Chen and S.-C. Lo, "Sliding-Mode Controller Design for Spacecraft Attitude Tracking Maneuvers," IEEE Transactions on Aerospace and Electronic Systems, vol. 29, no. 4, pp. 1328-1333, 1993.

[8] R. Qi, W. Su and Y. Meng, "Fault-Tolerant Attitude Controller Design for Deep Space Probe Via Adaptive Fast Terminal Sliding Mode Control," Journal of Dynamic Systems, Measurement, and Control, vol. 141, no. 9, 2019.

[9] S. Tashakori, S. Kasiri Bidhendi, B. Mashadi and J. Marzbanrad, "Trajectory Control and Sensitivity Analysis of Curiosity Rover on Uneven Terrains," Journal of Dynamic Systems, Measurement, and Control, vol. 141, no. 11, 2019.

[10] H. Bang, J.-s. Lee and Y.-j. Eun, "Nonlinear Attitude Control for a Rigid Spacecraft by Feedback Linearization," KSME International Journal, vol. 18, no. 2, pp. 203-2010, 2004.

[11] A. Giuseppi, A. Pietrabissa, S. Cilione and G. Luigi, "Feedback linearization-based satellite attitude control with a life-support device without communications," Control Engineering Practice, vol. 90, no. January, pp. 221-230, 2019.

[12] M. Navabi and M. Hosseini, "Spacecraft quaternion based attitude input-output feedback linearization control using reaction wheels," in International Conference on Recent Advances in Space Technologies, 2017.

[13] K. Godard, "Fault Tolerant Control of Spacecraft," 2010.

[14] M. J. Sidi, Spacecraft Dynamics and Control: A Practical Engineering Approach, New York: Cambridge University Press, 1981.

[15] L. Jonsson, "Simulations of Satellite Attitude Maneuvers - Detumbling and Pointing," 2019. 